\section{Regards sur l'économie allemande}

Bulletin économique du CIRAC

$66 \mid 2004$

Varia

\title{
Presse quotidienne : de la crise à la consolidation
}

Isabelle Bourgeois

\section{OpenEdition}

Journals

Édition électronique

URL : http://journals.openedition.org/rea/3868

DOI : $10.4000 /$ rea.3868

ISBN : 978-2-8218-0828-7

ISSN : 1965-0787

Éditeur

CIRAC

Édition imprimée

Date de publication : 1 mai 2004

Pagination : 37-39

ISSN : 1156-8992

Référence électronique

Isabelle Bourgeois, "Presse quotidienne : de la crise à la consolidation », Regards sur l'économie allemande [En ligne], 66 | mai 2004, mis en ligne le 13 octobre 2009, consulté le 15 septembre 2020. URL : http://journals.openedition.org/rea/3868 


\section{Actualité économique}

\section{PRESSE QUOTIDIENNE : de la crise à la consolidation}

Le quotidien suprarégional Frankfurter Rundschau vient d'être sauvé de la faillite grâce au rachat par la holding médias du parti SPD. A Berlin, le conflit sur la fusion des régionaux Der Tagesspiegel et Berliner Zeitung couve toujours - en attendant une réforme du droit des concentrations spécifique à la presse. Tous les quotidiens allemands sont en crise - du moins ceux qui sont diffusés essentiellement par abonnement, c'est-à-dire la majorité $(340$; diffusion payée : un peu plus de 20 millions d'exemplaires) des 380 titres publiés outre-Rhin. Leur CA total est tombé de quelque 11 milliards $€$ en 2000 à 9 milliards $€$ en 2003 .

Comme en France, leur lectorat diminue, surtout chez les jeunes qui lui préfèrent Internet ou les médias audiovisuels. Mais les 14-29 ans sont encore plus de $50 \%$ à lire un quotidien, rappelle la Fédération des éditeurs de presse Bundesverband Deutscher Zeitungsverleger (BDZV), et il s'agit moins d'un désintérêt pour l'information que d'un redéploiement vers d'autres sources d'information - celles dont l'offre s'est démultipliée au cours des 20 dernières années : les 250 radios, 30 chaînes gratuites (pour plus de 90 \% de la population) et Internet. Plus sensiblement qu'en France, la culture Internet a modifié aussi la structure des ressources de la presse quotidienne outre-Rhin.

Alors que traditionnellement, celle-ci tire ses revenus des ventes pour un tiers seulement, et aux deux tiers des annonces (publicité et offres emploi), elle voit s'effondrer ses recettes dans ce dernier domaine depuis 2000. La part des annonces dans le CA était alors encore de 64,4\%; deux ans plus tard, elle n'est plus que de $56,4 \%$, la tendance à la baisse se poursuivant en 2003. La part de la distribution dans le CA a crû en proportion, passant de $35,6 \%$ à 43,3 \%. Or parallèlement, les lecteurs, au budget de plus en plus serré, tendent à ne plus renouveler leur contrat avec l'un des deux quotidiens (un régional et un suprarégional) auxquels ils sont en moyenne abonnés. Les ventes reculent elles aussi.

Net recul du volume des annonces dans la presse quotidienne depuis 1997

\begin{tabular}{|lcccc|}
\hline (en milliers de $\mathrm{mm}$ ) & $\mathbf{1 9 9 7}$ & $\mathbf{2 0 0 0}$ & $\mathbf{2 0 0 3}$ ) & $\mathbf{2 0 0 3 / 1 9 9 7}$ \\
Total annonces & 1557764 & 1623306 & 1185000 & $-27 \%$ \\
dont : emploi & 185467 & 320836 & 100000 & $-46 \%$ \\
$\quad$ immobilier & 217903 & 192223 & 142000 & $-35 \%$ \\
$\quad$ automobile & 137353 & 134112 & 98000 & $-29 \%$ \\
\hline
\end{tabular}

Source des données : BDZV/ZMG/Ernst \& Young, Zeitungsverlage im Umbruch, 2003. *) Estimation.

Il est vrai que 2000 était une année record avec une forte croissance du PIB et donc un boom des offres d'emploi, mais aussi une intense activité publicitaire, dopée de surcroît par l'essor des services liés à la finance et aux TIC (où se recrutent pour l'essentiel les annonceurs des journaux). Cela relativise en un sens la chute du CA des quotidiens, par ailleurs mis à mal actuellement par le tassement général de la conjoncture comme du marché publicitaire. Le CA publicitaire des quotidiens est tombé à 4,93 milliards $€$ en $2002(-12,5 \%$ en un an), mais tous les supports ont enregistré un recul analogue, et les quotidiens maintiennent leur part de marché publicitaire $(26 \%$ en 2002 , après une hausse exceptionnelle à $29 \%$ en 2000). La 'crise' publicitaire les affecte donc peu. Il en va autrement des petites annonces, dont le volume (mesuré en $\mathrm{mm}$ ) accuse une tendance manifeste au recul : il a baissé de $27 \%$ en total, et même plus dans les secteurs-clef que sont l'immobilier, l'automobile et l'emploi.

Or cette baisse est de nature structurelle. Le repli continu des annonces immobilières n'est pas lié aux variations conjoncturelles, mais au lent retour à la normale d'un secteur hypertrophié par l'unification (" crise " du BTP) et l'expiration de diverses mesures fiscales d'incitation à l'investissement immobilier (voir $R E A$ 64/03). Le recul des annonces automobiles, généralement soumises à fluctuation, reflète plutôt en l'occurrence le déplacement des campagnes de promotion vers le support Internet.

Reste le cœur du problème: les offres d'emploi. Si on excepte l'année 2000, exceptionnelle par l'effervescence du marché de l'emploi (mutations liées à la " nouvelle économie "), et abstraction faite des fluctuations conjoncturelles, on observe un tendance lourde : la perte d'attractivité du support presse. Or la mobilité professionnelle n'a pas varié : plus de 7 millions d'Allemands changent d'emploi chaque année. C'est le mode de recrutement qui changé : il se fait de plus en plus souvent en ligne. Pour l'instant, cette tendance concerne surtout

\section{ANALYSE}

Montée en puissance de la culture Internet

Baisse des ventes et recul des annonces

Immobilier et automobile préfèrent Internet

La concurrence des offres d'emploi online... 
... met à mal le modèle économique..

... de la presse régionale surtout

Les éditeurs croient à une tendance conjoncturelle

La phase de consolidation engagée aboutira...

... à une offre suprarégionale de type premium,... les plus jeunes des actifs, où se recrutent aussi le plus d'internautes (voir REA 61/03), de même qu'elle se concentre encore sur le secteur tertiaire.

Mais la progression est inéluctable : non seulement, la 'fracture numérique' entre les âges tend à se réduire avec la rapide diffusion d'Internet, mais ce mode de communication s'impose jusque dans l'administration. Ainsi, l'Agence fédérale pour l'emploi offre désormais un service de recherche d'emploi en ligne ; et les postes proposés relèvent majoritairement du secteur secondaire. Parallèlement, un marché d'agences en ligne se constitue. C'est donc un des piliers de l'économie de la presse qui menace là de s'effondrer.

La presse régionale est plus affectée que les six quotidiens suprarégionaux : le volume des annonces a respectivement reculé de $-17 \%$ et $-37 \%$ entre 2000 et 2002, mais après avoir augmenté de $+2 \%$ seulement dans la PQR et de $+39 \%$ dans la presse suprarégionale entre 1997 et 2000. Or les éditeurs ne semblent pas avoir pris la réelle mesure de cette tendance, ainsi que le révèle une enquête réalisée par le cabinet Ernst \& Young en août 2003 auprès de 100 éditeurs de presse. Une bonne moitié d'entre eux $(55,5 \%)$ attribue le recul actuel des annonces à la récession, et les trois quarts $(73,7 \%)$ espèrent en conséquence une reprise dès le retour de la croissance.

Interrogés sur les principaux facteurs de "grand et/ou très grand " risque pour la branche, ils citent en majorité $(85,8 \%)$ la conjoncture, et en seconde position seulement la modification des habitudes de consommation des médias $(61,2 \%)$. Pour la moitié d'entre eux $(51,0 \%)$, ce sont ensuite les problèmes de financement, une crainte qui reflète le problème général des entreprises moyennes (les éditeurs allemands de presse quotidienne appartiennent presque tous au Mittelstand) confrontées aujourd'hui aux pratiques restrictives du crédit bancaire (voir dans ce numéro). Près de la moitié d'entre eux redoutent un phénomène de substitution médiatique $(45,9 \%)$ ou de concentration de l'édition $(44,9 \%)$. Ils sont nettement moins d'un tiers $(29,6 \%)$ à craindre la concurrence des journaux gratuits. Il est vrai que ce segment reste marginal outre-Rhin.

Les solutions envisagées pour sortir de la crise*

\begin{tabular}{|lccc|}
\hline & ...plutôt important & ...très important & Total \\
Compression des coûts & $37,8 \%$ & $56,1 \%$ & $\mathbf{9 3 , 9} \%$ \\
Coopération/partenariat & $48,0 \%$ & $38,8 \%$ & $\mathbf{8 6 , 8} \%$ \\
Développement de l'info locale & $25,5 \%$ & $57,1 \%$ & $\mathbf{8 2 , 6} \%$ \\
Elargir le lectorat & $37,8 \%$ & $41,8 \%$ & $\mathbf{7 9 , 6} \%$ \\
Innovation (optimisation des process) & $31,6 \%$ & $45,9 \%$ & $\mathbf{7 7 , 5} \%$ \\
Recentrage sur le cœur d'activité & $40,8 \%$ & $35,7 \%$ & $\mathbf{7 6 , 5} \%$ \\
Offre combinée papier/online & $38,8 \%$ & $22,4 \%$ & $\mathbf{6 1 , 2} \%$ \\
Plus de human interest & $38,8 \%$ & $19,4 \%$ & $\mathbf{5 8 , 2} \%$ \\
Diversification des supports & $33,7 \%$ & $17,3 \%$ & $\mathbf{5 1 , 0} \%$ \\
Plus d'infos sportives & $26,5 \%$ & $15,3 \%$ & $\mathbf{4 1 , 8} \%$ \\
Assouplir le droit des concentrations & $28,6 \%$ & $12,2 \%$ & $\mathbf{4 0 , 8} \%$ \\
\hline
\end{tabular}

Source : Ernst \& Young, Zeitungsverlage im Umbruch, 2003. *) Plusieurs réponses possibles.

Les solutions envisagées par les éditeurs sont, bien sûr, celles de toute PME confrontée à des difficultés (compression des coûts, recentrage, reconquête de la clientèle...). Elles sont également à la fois propres aux tendances générales du Mittelstand (coopération et partenariat) et spécifiques au secteur (media mix, accroissement de la part des thèmes porteurs dans le contenu rédactionnel). Mais ce sont là des réponses apportées au niveau de l'entreprise, non de la branche.

Or c'est l'environnement de la presse qui s'est totalement transformé en vingt ans : démultiplication de l'offre audiovisuelle dès 1984, extension du marché avec l'unification, extension des capacités de distribution (satellite et câble), libéralisation des télécommunications et rapide diffusion d'Internet. Ces mutations se sont traduites par une croissance strictement interne de l'ensemble des médias, c'est-à-dire sans capital industriel, ce qui contribue aux difficultés actuelles des éditeurs. Une phase de consolidation s'amorce ainsi depuis la fin des années 90 ; on en trouve le reflet dans la crainte exprimée par les éditeurs face au phénomène de concentration dans l'enquête du cabinet Ernst \& Young.

Ce dernier tente dès lors une esquisse du paysage de la presse dans dix ans. Premier type de quotidiens : les six titres suprarégionaux, comme la Frankfurter Allgemeine Zeitung (diffusion payée : 1,6 million d'exemplaires au total ; BDZV). Edités par de grandes PME et pourvus d'une forte identité de marque, ils occuperont le segment premium. Le produit papier, réservé presque exclusivement à la publicité institutionnelle et de marque, se doublera d'une plateforme de services électroniques avec une offre démultipliée d'annonces, et payante pour les non abonnés. Ces titres se financeront à l'avenir grâce à un prix de vente plus 
élevé, des recettes publicitaires accrues (version papier) et une gamme diversifiée de services en ligne payants. Ce concept est nouveau outre-Rhin, où la plupart des quotidiens offre encore gratuitement sur Internet l'accès à l'édition du jour (ou à une version électronique) - pour l'image exclusivement.

Second type de quotidiens : les régionaux, qui ont une diffusion payée de près de 16 millions d'exemplaires (titres locaux compris ; BDZV). Leur nombre se réduira, grâce à la fusion d'un certain nombre d'éditeurs - entreprises de taille moyenne. Il y aura grosso modo un titre par Land (c'est déjà le cas du Saarbrücker Zeitung en Sarre). Ils se concentreront sur l'information régionale, recourant à des pools de journalistes pour l'information nationale et étrangère (partenariat) ; les rédactions seront donc réduites, ce qui permettra de contenir les charges de personnel. Enfin, plus largement ouverts à la publicité régionale (hors tabac, il n'y a pas de secteurs interdits en Allemagne), ils pourront se vendre à un prix inférieur aux titres premium.

Troisième type : les quotidiens locaux (des petites PME) suivront deux évolutions selon leur zone de diffusion. Dans les régions densément peuplées, la plupart des titres a déjà été rachetée par les éditeurs de quotidiens régionaux (comme la Westdeutsche Allgemeine Zeitung dans la Ruhr, dont la société éditrice WAZ détient une large moitié des titres locaux diffusés en Rhénanie du Nord-Westphalie). Ceux-ci se contenteront d'un marché de niche. Dans les zones faiblement peuplées, à l'inverse, les petits quotidiens se maintiendront en réduisant les effectifs des rédactions et en créant des réseaux de partenariat.

Ce scénario se contente, en fait, de prolonger les évolutions qui s'observent sur le marché allemand de la presse quotidienne depuis la première grande vague de consolidation qui a suivi le boom des années 50 (la presse allemande, d'abord sous contrôle allié, recouvrait sa souveraineté éditoriale en 1955) et s'est achevée vers 1975, motivant l'adoption en 1976 d'un régime spécifique du droit des concentrations - celui qui freine la consolidation actuelle. Le nombre de titres était alors tombé de plus de 500 en 1955 à 410 . Mais le nombre "d'unités rédactionnelles " (sortes de pools produisant les diverses éditions correspondant à un titre) passait de 225 à 121, le nombre d'éditions passant de 1483 à 1194 . Il est aujourd'hui (en 2001) de 1541 éditions, réalisées par 129 unités. Le paysage allemand de la presse est d'une remarquable stabilité en ce qui concerne la diversité des éditions et fortement concentré à l'échelle des sociétés éditrices.

L'évolution se fera donc à deux niveaux. D'un côté, celui d'un nouveau business model permettant d'assurer la rentabilité des titres dans un environnement dominé par Internet. De l'autre, la poursuite de la concentration capitalistique au sein de la presse, mais aussi entre les divers médias (cross media ownership), ainsi que le montrent deux exemples. Le plus ancien est donné par le groupe WAZ (Essen), présent au tour de table de RTL Group. Le second est donné en ce moment par le groupe von Holtzbrink (PQR, radio, magazines), éditeur du quotidien économique suprarégional Handelsblatt et du titre régional Tagesspiegel : il cherche à racheter à Gruner + Jahr (Bertelsmann) la société éditrice du quotidien Berliner Zeitung - mais se heurte au veto de l'Office fédéral des Cartels.

La nécessaire consolidation du marché de la presse ne pourra se faire qu'après l'assouplissement de la réglementation du droit de la concentration propre à la presse. Actuellement, toute prise de participation est soumise à l'approbation de l'Office fédéral des Cartels dès lors qu'elle porte sur $25 \%$ des parts et aboutit à un CA cumulé de 25 millions $€$ par an. Cette disposition (avec ses seuils inférieurs au régime général national et européen), adoptée pour préserver le pluralisme des opinions à une époque où on redoutait un monopole de la presse Springer, est aujourd'hui le principal frein à la croissance économique du secteur. La fin de ce régime d'exception est certes programmée par l'intégration européenne, et la Fédération allemande des éditeurs de presse BDZV partage les grandes orientations du projet de loi de réforme du gouvernement fédéral.

Mais la modernisation est délicate à opérer puisqu'il s'agit, ni plus ni moins, de concilier droit constitutionnel et droit de la concurrence. La presse, outre-Rhin, est certes une activité économique (édition), mais elle jouit d'une garantie constitutionnelle (information) qui la soustrait au droit commun. La première vague de consolidation pouvait s'en accommoder: après l'expérience totalitaire de fraîche mémoire, l'appréciation du secteur ne pouvait se faire qu'en termes de liberté d'information ; en outre, la presse n'affrontait pas encore la concurrence d'un marché audiovisuel et elle était de surcroît un media confiné dans les frontières nationales. La seconde vague, qui se profile aujourd'hui, s'effectue dans le contexte d'un marché des médias, par ailleurs européanisé de facto - du moins en ce qui concerne les structures capitalistiques des plus grands acteurs - et où l'essor d'Internet a radicalement modifié les lois de la demande. Aux éditeurs et législateurs d'y adapter maintenant l'offre et le cadre réglementaire. (IB)
... une réduction du nombre de titres régionaux...

\section{... et une offre locale} de niche

Concentration du capital et offre diversifiée depuis 1975

Vers un nouveau business model et des liens cross media...

... après modernisation du droit de la concentration de la presse

Concilier droit constitutionnel et droit de la concurrence 